\section{A perfect functional marker for the gene of intermediate amylose content $W x$-in in rice (Oryza sativa L.)}

\section{Lei Zhou ${ }^{1 *}$, Shaoyu Chen ${ }^{1}$, Guocai Yang ${ }^{1}$, Wenjun Zha ${ }^{1}$, Haiya Cai ${ }^{1}$, Sanhe $\mathrm{Li}^{1}$, Zhijun Chen ${ }^{1}$, Kai Liu ${ }^{1}$, Huashan $\mathrm{Xu}^{1}$ and Aiqing You ${ }^{1,2}$}

\begin{abstract}
The amylose content $(A C)$ of rice is a key determinant of the grain cooking and eating quality. The Waxy $(W x)$ gene, which encodes granulebound starch synthase I (GBSSI), is the major gene controlling AC in rice. The $A$ - $C$ substitution in exon $6(E x 6 A / C)$ alters the amino acid codon from serine to tyrosine and is associated with intermediate-AC rice (Wx-in). In this paper, we reported a perfect functional marker for the SNP Ex6A/C of Wx-in based on polymerase chain reaction with confronting two-pair primers (PCR-CTPP) in a single-tube PCR assay. Specificity and applicability of the PCR assay were verified in a Chinese mini core collection (MCC) of Oryza sativa L. and a breeding population. We recommend the use of the simple, inexpensive assay for routine genotyping for intermediate $A C$ in the breeding population or discrimination of $W x$-in genotypes in rice germplasm.
\end{abstract}

Key words: Intermediate amylose content, waxy gene, marker-assisted selection, grain quality, rice.

\section{INTRODUCTION}

Rice is the most prominent cereal crop and staple food for more than half of the world's population (Normile 2008). After grain yield, quality is one of the most important aspects of rice breeding. Rice grain consists of approximately $90 \%$ starch, and amylose content (AC) is the key determinant of its cooking and eating quality. Previous studies indicated the rice gene $W x$ (waxy gene), which encodes a granule-bound starch synthase (GBSS), as a major gene controlling AC in endosperm starch (Tian et al. 2009, Wang et al. 1990), and AC was genetically mapped to the $W x$ gene (Wang et al. 2007). Four main functional alleles are found at the waxy locus of rice cultivars: $W x-b, W x-i n, W x-a$, and $w x$, corresponding to high $\mathrm{AC}$, intermediate $\mathrm{AC}$, low $\mathrm{AC}$, and waxy phenotype, respectively (Chen et al. 2008).

Moreover, two main functional single-nucleotide polymorphisms (SNPs) in the $W x$ gene have been identified, strongly associated with the AC and pasting properties of starch (Chen et al. 2010, Larkin and Park 2003, Ayres et al. 1997). The first SNP is the G/T SNP at the $5^{\prime}$ splice site of the first intron $(W x \ln 1)$. The $G$ - T substitution of $W x-a$ resulted in inefficient splicing of the RNA transcript and decreased production of mature mRNA, thus reflecting a low AC (Cai et al. 1998). The second SNP is an A - C substitution in exon 6 of $W x$-in (Ex6A/C), which alters the amino acid codon from serine to tyrosine and is associated with
Crop Breeding and Applied Biotechnology 18: $103-109,2018$ Brazilian Society of Plant Breeding. Printed in Brazil http://dx.doi.org/10.1590/198470332018v18n1a14

70332018v18n1a14

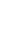




\section{Zhou et al.}

intermediate-AC rice (Chen et al. 2010, 2008). Cooked rice with low AC is sticky, little swelled, soft, and glossy; cooked rice with high AC is easily retrogradable, dry, hard, and dull after cooling; while cooked intermediate-AC rice is fluffy and soft, non-retrogradable, and has high palatability. Due to these qualities, the latter is the most popular rice type in China and an important indicator of the national standard of high-quality rice (GB/T17891-1999) (Standardization Administration of the People's Republic of China, 2000). Therefore, rice breeding has been focused on the development of varieties with the desired intermediate $A C$ and excellent overall performance to meet the standards of special end products.

Functional Markers (FMs) developed from functional gene sequences accurately discriminate the alleles at a single locus and represent ideal markers for marker-assisted selection (MAS) in breeding. The FMs have apparent advantages over random DNA markers, because they are fully diagnostic of the target trait allele (Varshney et al. 2005). Genetic linkage between a specific linked marker and a target locus allele, identified by QTL studies, for example, can be broken by genetic recombination; this limits the use of linked markers as a diagnostic tool (Andersen and Lübberstedt 2003). As a result, relatively few linked markers are actually used in breeding. So far, multiple SNP typing methods including sequencing, mass-spectrography, high resolution melting, SNP chip, SNaPshot, etc. are being employed (Daniel et al. 2015, Yu et al. 2014, Li et al. 2010, Masouleh et al. 2009), although these methods are technically complex, time-consuming and expensive for large-scale screening in breeding programs. The derived Cleaved Amplified Polymorphic Sequence (dCAPS) is a relatively simple method used for SNP detection (Neff et al. 1998), but requires time-consuming postamplification enzymatic cleavage. Polymerase chain reaction with confronting two-pair primers (PCR-CTPP) is another method for SNP typing. It requires four primers with single PCR reaction and the SNP type is determined according to the product length, making it the best choice for high-throughput SNP typing (Hamajima 2001). In this study, we attempted to develop a simple, codominant functional marker targeting $W x$-in that can be resolved on simple agarose gels and is easy to use for MAS for intermediate AC involving large breeding populations.

\section{MATERIAL AND METHODS}

\section{Plant material}

Two hundred and sixty cultivated rice varieties of the Chinese mini core collection (MCC) of Oryza sativa L. were grown in the field in Wuchang, Hubei province, China, using the cultural management practices recommended for the region. The MCC is composed of the most representative varieties, screened from more than 60000 accessions of Chinese cultivated rice, comprising $0.3 \%$ of the accessions in the basic collection, and retaining $70.65 \%$ of the SSR variation and 76.97\% of the phenotypic variation (Zhang et al. 2011).

\section{DNA Extraction}

DNA was extracted from leaves following the CTAB method (Rogers and Bendich 1988), with minor modifications.

\section{Genotyping for Wx gene}

\section{SNP $W x-\ln 1$}

The marker, specific for the SNP Wx-In1 developed by Cai et al. (2015), was used as the functional marker for genotyping the $W x$ gene of the mini core collection.

\section{SNP Wx-Ex6}

Functional markers for Wx-Ex6 confronting two-pair primers were designed based on the PCR-CTPP method. Primers were designed according to the $W x$ sequence of Lemont (an elite variety in the USA with intermediate AC and $C$ base at target SNP Wx-Ex6) and 9311 (an elite variety in China with A base at target SNP). For A-type allele-specific primers (Figure 1), Ex6AR was first fixed upstream of target SNP loci (A base) together with its complementary base as $3^{\prime}$-end, using the manual search mode of Primer Premier 5.0, and then the $5^{\prime}$-terminal loci of Ex6AR was fixed with edit primer function to analyze its melting temperature $(\mathrm{Tm})$ until it was about $60^{\circ} \mathrm{C}$. There was no limitation for the location of Ex6AF; a primer sequence of Ex6AF $\left(\operatorname{Tm} \sim 55^{\circ} \mathrm{C}\right)$ matching Ex6AR with highest score was selected using the automatic search mode. The C-type allele specific primers Ex6CF and Ex6CR were developed in the same way. 


\section{PCR amplification}

A $15 \mathrm{~mL}$ reaction mixture containing $1.5 \mathrm{~mL}$ of PCR buffer $(20 \mathrm{mM}$ Tris, $\mathrm{pH} 8.0,50 \mathrm{mM} \mathrm{KCl}, 2.5 \mathrm{mM} \mathrm{MgCl}, 0.1 \mathrm{mM}$ EDTA, $1 \mathrm{mM}$ DTT, $50 \%$ glycerol), $50 \mathrm{ng}$ of DNA, $330 \mathrm{nM}$ of four primers each, $250 \mathrm{mM}$ of each dNTP, and 1.0 units of Taq polymerase was used for PCR amplification. The samples were prepared in a 96-well amplification plate using a MyCycler Thermal Cycler System (Bio-Rad, USA). The PCR conditions were $95{ }^{\circ} \mathrm{C}$ for $5 \mathrm{~min} ; 35$ cycles of $95{ }^{\circ} \mathrm{C}$ for $30 \mathrm{~s} ; 55^{\circ} \mathrm{C}$ for $30 \mathrm{~s} ; 72{ }^{\circ} \mathrm{C}$ for $45 \mathrm{~s}$; and $72{ }^{\circ} \mathrm{C}$ for $5 \mathrm{~min}$. The amplified PCR products were separated on $1.5 \%$ agarose gels (Amresco, USA) stained with ethidium bromide and visualized using GelDocXR (Bio-Rad, USA). The gels were scored based on the banding pattern, as positive, negative and heterozygous.

\section{Application of the functional marker in a breeding population}

A breeding population $\left(F_{6}\right.$, Jufeng $B / /$ Tianfeng $\left.B / J 521\right)$, developed by multiple crosses followed by selfing for five generations to $F_{6}$, was used to validate the $W x$-in functional marker. J521 was a bred line with intermediate $A C$ and the $W x$-in gene from the wide compatibility variety Lemont. Jufeng $B(W x-b)$ and Tianfeng $B(W x-a)$ were two elite threeline hybrids, maintainer lines, one with low and the other high AC, respectively. In the selfing generations, plants with good agronomic traits and high yield were visually selected.

\section{Determination of amylose content}

Mature seeds were harvested and threshed by hand; the grains were dehulled, and all broken, diseased and immature kernels were manually removed. The AC was determined with procedures described by GB/T 15683-2008/ISO 6647-1 including boiling, cooling, ether extraction, and titration (Standardization Administration of the People's Republic of China 2008).

\section{RESULTS}

\section{Development of marker for functional SNP Wx-Ex6}

Confronting two-pair primers were designed based on the PCR-CTPP method. According to the PCR-CTPP theory, the mismatch at 3'-end between Ex6AR and C-type template (T/C) or Ex6CF and A-type template (C/T) would interrupt amplification when the mixture of four primers was amplified separately with A-type or C-type template. Nevertheless, our results showed that one mismatch could not interrupt amplification of the allele-specific primer with template, so another mismatch (G/G, A/A) was introduced at -3 ' loci of Ex6AR and Ex6CF to strengthen its specificity (Figure 1). Details of the primers were listed in Table 1 . We successfully developed the codominant PCR-CTPP markers to genotype the functional SNP Wx-Ex6 in the rice $W x$ gene. With the method described in this study, SNP typing of rice Wx-Ex6 can be achieved easily.

\section{Correlation analysis between AC and haplotype of WxIn1-Ex6 in the MCC of Oryza sativa L. in China}

In order to further test the specificity of Wx-Ex6, we examined the haplotype distribution of $W x \ln 1$-Ex6 in the MCC of Oryza sativa L. in China. The AC values of 249 accessions in the MCC were determined, and 31 waxy rice accessions were found with a $A C$ value lower than $2.0 \%$. Among the 218 non-waxy rice accessions, there were three haplotypes of $W x \ln 1-E x 6$ (In1G-Ex6A, In1G-Ex6C, In1T-Ex6A), 130 accessions had the haplotype of In1G-Ex6A with a mean AC of $23.4 \%$ (high), 21 accessions had the haplotype of In1G-Ex6C with a mean AC of 18.5\% (intermediate), and 67 accessions

Table 1. Primer details of WxEx6-specific marker

\begin{tabular}{lcc}
\hline Primer name & Primer sequence $\left(5^{\prime} \rightarrow 3^{\prime}\right)$ & $\begin{array}{c}\text { Product type } \\
\text { Product length } \\
(\mathrm{bp})\end{array}$ \\
\hline Ex6AF & CTGGAGAAGGTGGAGTCAT & \\
Ex6CR & GGCGGTGATGTACTTGTCC & AF-CR (Common) band) \\
Ex6AR & GATCTTGAGATCAATTGTAACTCACGAT & AF-AR (A type) \\
Ex6CF & CAACCCATACTTCAAAGGAACATC & CF-CR (C type) \\
& & 310 \\
\hline
\end{tabular}

Underlined nucleotide: mismatched base; bold font nucleotide: functional SNP 
had the haplotype of In1T-Ex6A with a mean AC of $14.0 \%$ (low), while haplotype of In1T-Ex6C was not detected (Figure 2). According to correlation analysis and regression analysis using Microsoft EXCEL, the correlation coefficient between haplotype and $A C$ value was 0.93 , the $P$ value of regression analysis was $<0.001$, and the haplotype of In1-Ex6 explained $86.8 \%$ of total AC variation. These two properties showed an extremely significant positive correlation between genotypes and phenotypes, indicating that the haplotype of In1-Ex6 can be used to identify AC accurately. Of the haplotypes of $W x \ln 1-E x 6$ in the Chinese mini core collection of Oryza sativa L., only intermediate AC gene $W x$-in (In1G-Ex6C) had the SNP Ex6C genotype in non-waxy rice. Hence, the functional codominant marker for SNP Ex6C developed in this study can be used to screen intermediate $\mathrm{AC}$ gene $W x$-in (In1G-Ex6C) in non-waxy rice.

\section{Application of the functional marker in breeding populations}

The $F_{6}$ breeding population including 192 lines were genotyped with the functional marker for Wx-Ex6 confronting two-pair primers. By the genotypic analysis of the $F_{6}$ breeding population segregated at the target SNP loci with $W x$-Ex6 specific marker, $48 W x$-in positive lines were detected, including 43 homozygous lines (CC) and 5 heterozygous lines (CA) (Figure 3 ). The AC values of 48 positive lines and 22 negative lines $(A A)$ were measured. The AC value of $\mathrm{W} x$-in positive lines ranged from 16.2 to $21.9 \%$ (average 17.6\%), all belonging to the intermediate-AC rice group (Figure 4). The AC values of the 22 negative lines (AA) can be divided in two types, i.e., higher than $22 \%$ and lower than $15 \%$, corresponding to high-AC rice (In1G-Ex6A) and low-AC rice (In1T-Ex6A), respectively. The significance test for $A C$ values indicated extremely significant differences ( $p$ $<0.001$ ) between CC/CA and AA types, and no significant difference between $C C$ and CA types. The verification test indicated that the positive marker performance was highly consistent with the phenotypic performance, suggesting that the codominant marker for functional SNP Wx-Ex6C developed in this study can be used to screen intermediate$A C$ rice accurately.

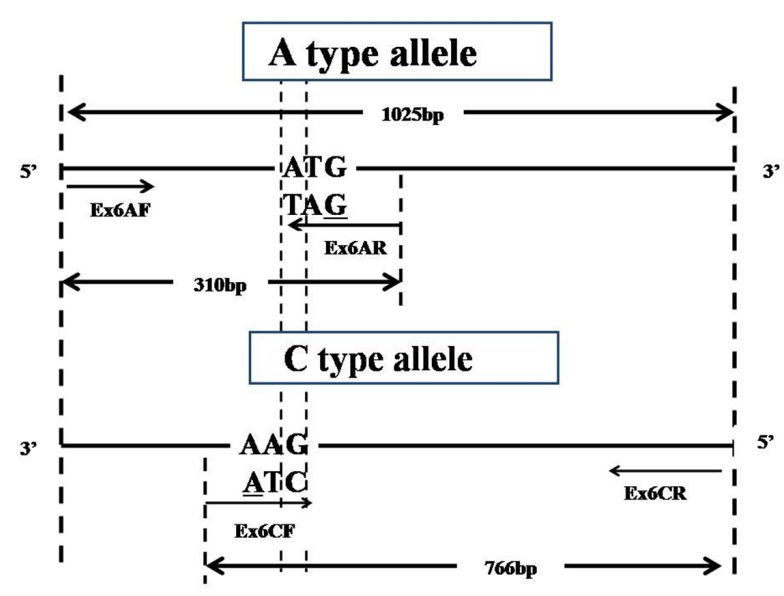

Figure 1. Schematic representation of SNP typing method for rice WxEx6 based on PCR with confronting two-pair primers (PCRCTPP). Target SNP loci is A/C substitution, mismatch between 3 ' end base, -3 loci of Ex6CF and Ex6AR and template ensured allele specificity. Both alleles have a $310 \mathrm{bp}$ product for A-type allele, a 766 bp product for C-type allele and a 1025 bp product in common.

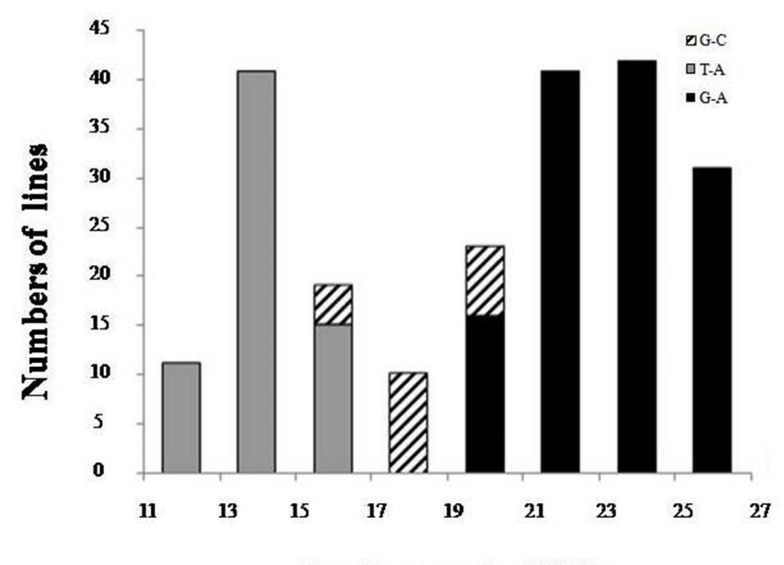

Amylose content (\%)

Figure2. The relationship between amylose content and the haplotype of $W x-(\ln 1-\mathrm{Ex} 6)$ in 218 non-waxy rice accessions in the MCC of Oryza sativa L.in China.

\section{DISCUSSION}

Improving the rice quality is of great significance for both rice producers and consumers. The eating and cooking quality of rice mainly relies on amylose content (AC), gel consistency (GC), and gelatinization temperature (GT). All these factors are correlated with each other, and genes related to starch synthesis collectively form a fine regulating network that controls the eating and cooking quality and defines the correlations among the three properties (Tian et al. 2009). In this study, more than 200 core germplasms revealed abundant genetic variation of AC, ranging from 1 to $27 \%$, which would provide rich germplasm resources for rice with improved AC. Currently, the AC of most rice varieties in China is either high or low, and breeding rice varieties with intermediate AC will be of great worth. 


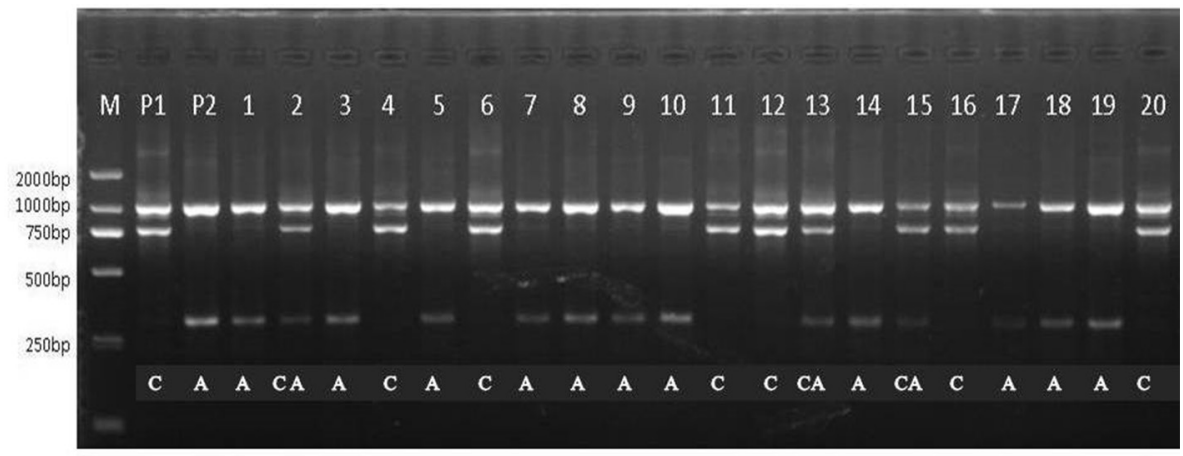

Figure 3. Genotypic analysis of the $\mathrm{F}_{6}$ breeding population segregated at target SNP loci with Wx-Ex6 specific marker. M: DL2000 DNA marker. P1 and P2 are the check varieties Lemont and 9311, which belong to C-type and A-type respectively; lanes 1-20 are parts of $F_{6}$ lines and the SNP type of each plant is presented under the corresponding lane.

Of the haplotypes of $W x \ln 1-E x 6$ in the Chinese mini core collection of Oryza sativa L., the SNP Ex6C genotype was detected exclusively in the haplotype of the intermediateAC gene $W x$-in (In1G-Ex6C) in non-waxy rice, which was consistent with previous studies (Chen et al. 2008, Larkin and Park 2003). Therefore, the co-dominant marker for functional SNP Ex6C developed in this study can be used to screen the intermediate- $A C$ gene $W x$-in in non-waxy rice. The functional marker for SNP Ex6A/C contained two allele-specific primers and one common primer labeled with different fluorescence probes was developed by the AS-PCR method. The PCR products were analyzed by a capillary electrophoresis system (Chen et al. 2010), which is technically complex, and rather expensive for most Chinese breeding teams for large-scale screening in breeding programs. In this study, a four-primer codominant marker designed by the PCR-CTPP method from functional SNP Ex6A/C of $W x$-in on $1.5 \%$ agarose gel ensured rapid and

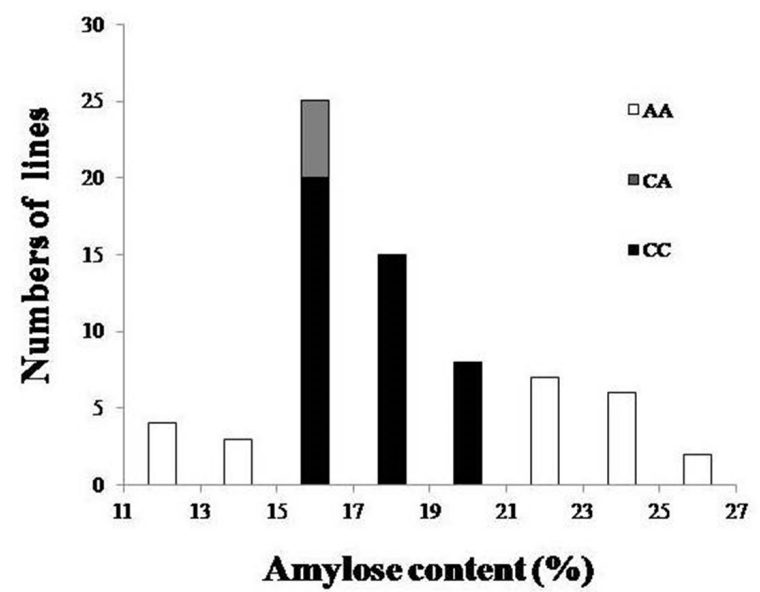

Figure4. Amylose content distribution of the $\mathrm{F}_{6}$ breeding population with three types alleles of SNP Wx-Ex6(AA/CC/CA). accurate classification. Two verification tests indicated that the positive marker performance was concomitant with the phenotypic performance. Therefore, the codominant marker for functional SNP Ex6A/C developed in this study can be used to accurately screen non-waxy rice with intermediate AC. Likewise, the simple, codominant functional marker would facilitate MAS for $W x$-in involving large breeding populations and germplasm screening. In this study, 31 waxy rice accessions in the MCC were genotyped by the SNP markers viz., Wx-In1 and Wx-Ex6. The accession numbers with the haplotypes In1T-Ex6A, In1T-Ex6C and In1G-Ex6A were 25, 2 and 3 respectively, and there was no In1G-Ex6C haplotype in the waxy varieties. The waxy phenotype cannot be selected using SNP markers, because the waxy phenotype may be caused by several other mutated loci of Waxy (Inukai et al. 2000). However, this phenotype is often and commonly detected visually in breeding programs.

Anonymous molecular markers were traditionally used in MAS for resistance or other excellence traits (Hittalmani et al. 2000, Huang et al. 1997). However, even for tightly linked markers, the effectiveness of MAS is greatly diminished by the occasional uncoupling of the marker from the trait during the numerous meiotic cycles in breeding programs, which may induce errors in selection of the desired traits (Tian et al. 2016, Perumalsamy et al. 2010). Hence, the use of functional markers (FMs), derived from polymorphic sites within gene sequences affecting phenotypic variation, is more efficient for gene identification and selection. With the availability of information on cloned resistance genes, the development of FMs will become common in the future (Zhou et al. 2013, Bradbury et al. 2005, Andersen and Lübberstedt 
2003). Reduced breeding costs, simple operation, and short cycles are the advantages of MAS, which seems to have a bright future for application in rice breeding (Tian et al. 2016, Andersen and Lübberstedt 2003). The PCR products for $W x$-in in this study can be analyzed easily and inexpensively on agarose gel or alternatively with more sophisticated high throughput equipment, making PCR assays a very versatile tool.

\section{ACKNOWLEDGEMENTS}

We thank Dr. Saddam Hussain and Miss Cornelia Abreu for revision of English in the manuscript. This work was funded by the National High Technology Research and Development Program of China (2014AA10A604), the Major State Basic Research Development Program of China (2013CBA01405), and the Key Project of Hubei Province Science Foundation of China (2015CFA159).

\section{REFERENCES}

Andersen JR and Lübberstedt T (2003) Functional markers in plants. Trends in Plant Science 8: 554-560.

Ayres NM, McClung AM, Larkin PD, Bligh HFJ, Jones CA and Park WD (1997) Microsatellites and a single-nucleotide polymorphism differentiate apparent amylose classes in an extended pedigree of US rice germplasm. Theoretical and Applied Genetics 94: 773-781.

Bradbury LMT, Henry RJ, Jin QS, Reinke RF and Waters DLE (2005) A perfect marker for fragrance genotyping in rice. Molecular Breeding 16: $279-283$.

Cai HY, Xu DZ, Zhou L, Cheng JP, Zhang ZH, Wu JP and You AQ (2015) Development of PCR-based SNP marker of rice Waxy gene with confronting two-pair primers. Russian Journal of Genetics 51: 787-791.

Cai X, Wang Z, Xing Y, Zhang J and Hong M (1998) Aberrant splicing of intron 1 leads to the heterogeneous 5' UTR and decreased expression of waxy gene in rice cultivars of intermediate amylose content. Plant Journal 14: 459-465.

Chen M, Bergman C, Pinson S and Fjellstrom R (2008) Waxy gene haplotypes: associations with apparent amylose content and the effect by the environment in an international rice germplasm collection. Journal of Cereal Science 47: 536-545.

Chen M, Fjellstrom RG, Christensen EF and Bergman CJ (2010) Development of three allele-specific codominant rice Waxy gene PCR markers suitable for marker-assisted selection of amylose content and paste viscosity. Molecular Breeding 26: 513-523.

Daniel R, Santos C, Phillips C, Fondevil M, Oorschot RAH, Carracedo A, Lareu MV and McNevin D (2015) A SNaPshot of next generation sequencing for forensic SNP analysis. Forensic Science International: Genetics 14: 50-60.

Hamajima N (2001) PCR-CTPP: a new genotyping technique in the era of genetic epidemiology. Expert Review of Molecular Diagnostics 1: $119-123$.

Hittalmani S, Parco A, Mew TV, Zeigler RS and Huang N (2000) Fine mapping and DNA marker-assisted pyramiding of the three major genes for blast resistance in rice. Theoretical and Applied Genetics 100: 1121-1128.
Huang N, Angeles ER, Domingo J, Magpantay G, Singh S, Zhang G, Kumaravadivel N, Bennett J and Khush GS (1997) Pyramiding of bacterial blight resistance genes in rice: marker-assisted selection using RFLP and PCR. Theoretical and Applied Genetics 95: 313-320.

Inukai T, Sako A, Hirano H and Sano Y (2000) Analysis of intragenic recombination at wx in rice: correlation between the molecular and genetic maps within the locus. Genome 43: 589-596.

Larkin P and Park W (2003) Association of waxy gene single-nucleotide polymorphisms with starch characteristics in rice (Oryza sativa L.). Trends in Plant Science 12: 335-339.

Li YD, Chu ZZ, Liu XG, Jing HC, Liu YG and Hao DY (2010) A cost-effective high-resolution melting approach using the EvaGreen dye for DNA polymorphism detection and genotyping in plants. Journal of Integrative Plant Biology 52: 1036-1042.

Masouleh AK, Waters DLE, Reinke RF and Henry RJ (2009) A highthroughput assay for rapid and simultaneous analysis of perfect markers for important quality and agronomic traits in rice using multiplexed MALDI-TOF mass spectrometry. Plant Biotechnology Journal 7: 355-363.

Neff M, Neff J and Chory J (1998) dCAPS, a simple technique for the genetic analysis of single-nucleotide polymorphisms: experimental applications in Arabidopsis thaliana genetics. Plant Journal 14: 387-392.

Normile D (2008) Reinventing rice to feed the world. Science 321: 330-333.

Perumalsamy S, Bharani M, Sudha M, Nagarajan P, Arul L, Saraswathi R, Balasubramanian P and Ramalingam J (2010) Functional markerassisted selection for bacterial leaf blight resistance genes in rice (Oryza sativa L.). Plant Breeding 129: 400-406.

Rogers OS and Bendich AJ (1988) Extraction of DNA from plant tissues. Plant Molecular Biology Manual A6: 1-10.

Standardization Administration of the People's Republic of China (2008) GB/T15683-2008/ISO 6647-1:2007 Rice-determination of amylose content. Standard Press, Beijing, p. 1-16

Standardization Administration of the People's Republic of China (2000) GB/T17891-1999 High quality paddy. Standard Press, Beijing, p. 1-6

Tian DG, Chen ZJ, Chen ZQ, Zhou YC, Wang ZH, Wang F and Chen SB (2016) Allele-specific marker-based assessment revealed that the rice 
A perfect functional marker for the gene of intermediate amylose content $W x$-in in rice (Oryza sativa L.)

blast resistance genes Pi2 and Pi9 have not been widely deployed in Chinese indica rice cultivars. Rice 9: 19.

Tian ZX, Qian Q, Liu QQ, Yan MX, Liu XF, Yan CJ, Liu GF, Gao ZY, Tang SZ, Zeng DL, Wang YH, Yu JM, Gu MH and Li JY (2009) Allelic diversities in rice starch biosynthesis lead to a diverse array of rice eating and cooking qualities. Proceedings of the National Academy of Sciences of the USA 106: 21760-21765.

Varshney RK, Graner A and Sorrells ME (2005) Genomics-assisted breeding for crop improvement. Trends in Plant Science 10: 621-630.

Wang L, Liu W, Xu Y, He Y, Luo L, Xing Y, Xu C and Zhang Q (2007) Genetic basis of 17 traits and viscosity parameters characterizing the eating and cooking quality of rice grain. Theoretical and Applied Genetics 115: 463-476.

Wang ZY, Zheng FQ, Shen GZ, Gao JP, Snustad DP, Li MG, Zhang JL and Hong MM (1995) The amylose content in rice endosperm is related to the post-transcriptional regulation of the waxy gene. Plant Journal 7: 613-622.

Wang ZY, Wu ZL, Xing YY, Zheng FG, Guo XL, Zhang WG and Hong MM (1990) Nucleotide sequence of rice waxy gene. Nucleic Acids Research 18: 5898.

Yu HH, Xie WB, Li J, Zhou FS and Zhang QF (2014) A whole-genome SNP array (RICE6K) for genomic breeding in rice. Plant Biotechnology Journal 12: 28-37.

Zhang HL, Zhang DL, Wang MX, Sun JL, Qi YW, Li JJ, Wei XH, Han LZ, Qiu ZE, Tang SX and Li ZC (2011) A core collection and mini core collection of Oryza sativa L. in China. Theoretical and Applied Genetics 122: 49-61.

Zhou L, Chen ZJ, Lang XY, Du B, Liu K, Yang GC, Hu G, Li SH, He GC and You AQ (2013) Development and validation of a PCR-based functional marker system for the brown planthopper resistance gene Bph14 in rice. Breeding Science 63: 347-352. 